\title{
Human adipose-derived mesenchymal stem cell spheroids improve recovery in a mouse model of elastase-induced emphysema
}

\author{
Ryeon Jin Cho, ${ }^{1, \#}$,You-Sun Kim ${ }^{1,2, \#}$, Ji-Young Kim ${ }^{2} \mathcal{E}$ Yeon-Mok Oh ${ }^{1,2,3, *}$ \\ ${ }^{1}$ University of Ulsan College of Medicine, Seoul 05505, ${ }^{2}$ Asan Institute for Life Science, Seoul 05505, ${ }^{3}$ Department of Pulmonary and \\ Critical Care Medicine, and Clinical Research Center for Chronic Obstructive Airway Disease, Asan Medical Center, Seoul 05505, Korea
}

\begin{abstract}
Emphysema, a pathologic component of the chronic obstructive pulmonary disease, causes irreversible destruction of lung. Many researchers have reported that mesenchymal stem cells can regenerate lung tissue after emphysema. We evaluated if spheroid human adipose-derived mesenchymal stem cells (ASCs) showed greater regenerative effects than dissociated ASCs in mice with elastase-induced emphysema. ASCs were administered via an intrapleural route. Mice injected with spheroid ASCs showed improved regeneration of lung tissues, increased expression of growth factors such as fibroblast growth factor-2 (FGF2) and hepatocyte growth factor (HGF), and a reduction in proteases with an induction of protease inhibitors when compared with mice injected with dissociated ASCs. Our findings indicate that spheroid ASCs show better regeneration of lung tissues than dissociated ACSs in mice with elastase-induced emphysema. [BMB Reports 2017; 50(2): 79-84]
\end{abstract}

\section{INTRODUCTION}

Chronic obstructive pulmonary disease (COPD) is the fourthleading cause of death worldwide and includes cases of chronic bronchitis and emphysema. Emphysema is characterized by alveoli damaged by external factors, such as smoking (1). Matrix metalloproteases (MMPs) are extracellular matrix (ECM)-associated enzymes involved in the synthesis and degradation of connective tissue components for tissue remodeling and repair (2). A recent study determined that

*Corresponding author. Tel: +82-2-3010-3136; Fax: +82-2-30104650; E-mail: ymoh55@amc.seoul.kr

${ }^{\text {"T}}$ These authors contributed equally to this work.

https://doi.org/10.5483/BMBRep.2017.50.2.101

Received 16 June 2016, Revised 1 July 2016, Accepted 14 October 2016

Keywords: Adipose-derived mesenchymal stem cells, Chronic obstructive pulmonary disease, Regeneration, Spheroid cultures secreted MMPs (MMP-2, 9, and 12) contributed to the development of emphysema by degrading the alveolar wall matrix (3). In emphysema, regeneration of the alveolar wall is impaired; however, a mesenchymal stem cell (MSC) therapy for this condition is currently under development. MSCs that have been isolated from various organs, such as bone marrow, adipose tissue, and umbilical cord blood, can induce tissue repair via self-renewal, differentiation, and paracrine effects (4). The recovery effects of MSCs have been confirmed in various lung disease models, including lipopolysaccharide (LPS)-, cigarette smoke-, and bleomycin-induced lung injury models (5-7).

We previously tracked adipose-derived mesenchymal stem cells (ASCs) that were injected in vivo via intravenous routes. Labeled ASCs were detected for up to $24 \mathrm{~h}$ post-injection (8). Moreover, only $17 \%$ of cardiac sphere-derived stem cells survived up to $1 \mathrm{~h}$ after injection, and therapies using dissociated stem cells showed complications, including cell loss, in a myocardial infarction model $(9,10)$.

A spheroid is a self-assembled group of cells, and many studies have emphasized the differences between 2D and 3D culture environments, such as nutrient and oxygen gradients, cell-to-cell interactions, and matrix deposition $(11,12)$. A recent study showed that spheroids increase anti-apoptotic and anti-inflammatory properties both in vitro and in vivo (13). In this report, we used a mouse model of elastase-induced emphysema. Mice received intrapleural injections of aggregated "spheroid" ASCs to compare their therapeutic efficacy with that of dissociated ASCs.

\section{RESULTS}

Characterization of dissociated and spheroid ASCs

A monolayer of ASCs cultured for 3 days (Supplementary Fig. 1A) were seeded into PDMS- concave microwells coated with $3 \%$ BSA. Five minutes after seeding, unattached ASCs were removed using the ASC-conditioned medium. Cells began to aggregate within $1 \mathrm{~h}$ of seeding (Supplementary Fig. 1B). Generally speaking, homogeneous spheroid ASCs were observed after 24 h (Supplementary Fig. 1C). 
To characterize the dissociated and spheroid ASCs, we measured the expression of cell survival-related apoptosis proteins and growth factors in protein samples extracted from ASCs. BCl-2 inhibits interactions between mitochondria and $\mathrm{BAX}$ in the cytosol. The Western blot images suggested that BAX expression was similar in spheroid and dissociated ASCs, whereas $\mathrm{Bcl}-2$ expression was increased in spheroid ASCs (Fig. 1A). ImageJ was used to quantify protein levels. We determined that BAX expression was significantly decreased and BCL2 expression was significantly increased in spheroid ASCs when compared with dissociated ASCs. Furthermore, the $\mathrm{BCl}-2 / \mathrm{BAX}$ ratio was significantly increased in spheroid ASCs (Fig. 1B). FGF-2 and VEGF levels were also significantly increased in spheroid ASCs (Fig. 1C and D). To measure the levels of secreted growth factors, the culture medium of dissociated and spheroid ASCs was concentrated, and growth factors were measured by ELISA (Fig. 1F). VEGF secretion was increased in spheroid ASCs when compared with dissociated ASCs. FGF2 was not detected in the media from either group.

\section{Spheroid ASCs improved lung recovery in an elastase-induced mouse model of emphysema}

Emphysema was induced by elastase in C57BL/6 mice (day 0). After 7 days, mice were intrapleurally injected with $1 \times 10^{5}$ dissociated or spheroid ASCs. On day 14, mice were euthanized (Fig. 2A), and lung tissues were collected. The $\mathrm{H}$ and E-stained lungs of the elastase-treated group showed severe alveolar destruction (Fig. 2C) when compared with the control group (Fig. 2B). The mice treated with the two types of ASCs showed recovery from the alveolar damage (Fig. $2 \mathrm{D}$ and E). We used the mean linear intercept (MLI) method to quantify this recovery. The MLI was reduced in the dissociated ASC-infused group $(97.9 \mu \mathrm{m})$ and spheroid ASC-infused group $(78.2 \mu \mathrm{m})$ when compared with the elastase only group $(109.6 \mu \mathrm{m})$. The
A

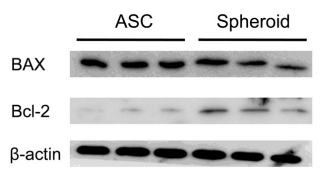

B

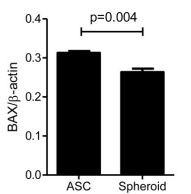

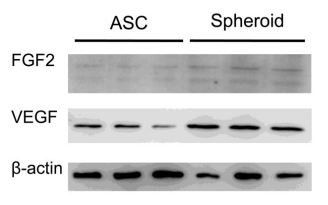

D

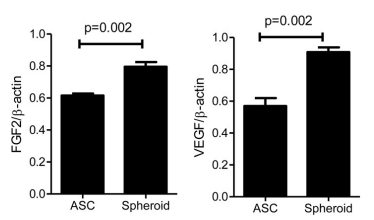

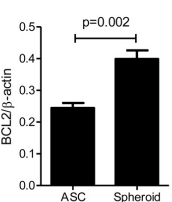

E

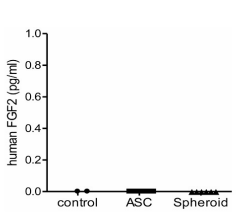

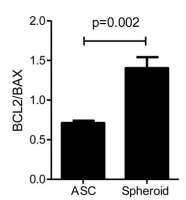

$\mathbf{F}$

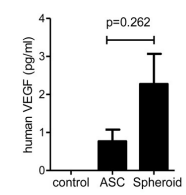

Fig. 1. The expression of apoptotic markers and growth factors. (A) Western blotting images and (B) protein band quantification for BAX and Bcl-2. (C) Western blotting images and (D) protein band quantification for FGF-2 and VEGF. (E) FGF-2 and (F) VEGF production was measured in conditioned media.

\section{A}
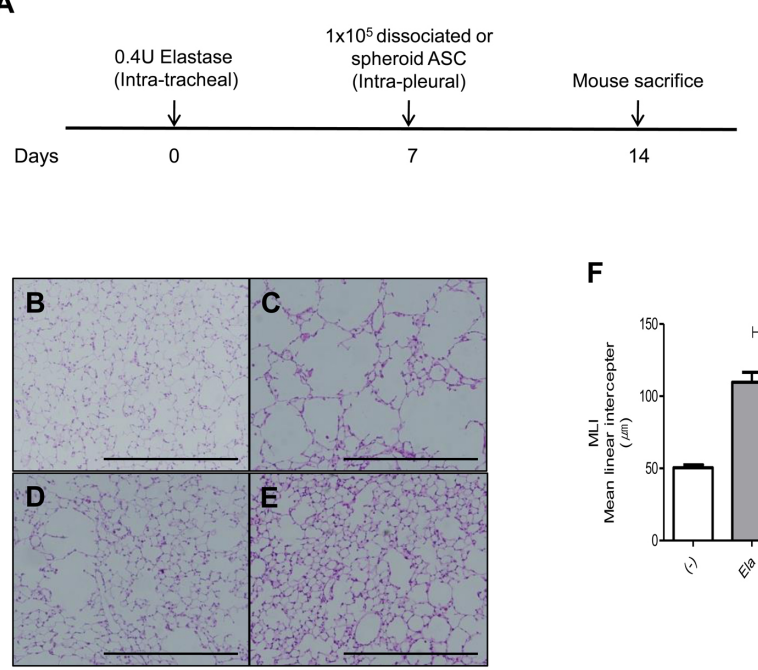

$\mathbf{F}$

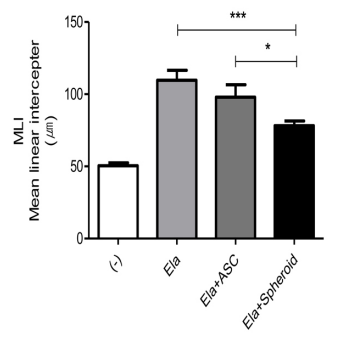

Fig. 2. Improved recovery efficacy of ASCs in an elastase-induced mouse model of emphysema. (A) Experimental protocol. (B-E) Lung histology with $\mathrm{H}$ and $\mathrm{E}$ staining: (B) control $(\mathrm{n}=5)$; (C) elastase only ( $\mathrm{n}=12)$; (D) elastase + dissociated ASCs $(\mathrm{n}=10)$; and (E) spheroid ASCs $(n=11)(10 \times)$. (F) Mean linear intercept (mean \pm SEM). Scale bar $=0.5 \mathrm{~mm}$. ${ }^{*} \mathrm{P}<0.05$ and $* * * P<0.001$ for comparisons between 2 groups. 
MLI of the spheroid ASC-infused group showed a stronger reduction than the dissociated ASC-infused group. These findings suggest that the recovery between the dissociated and spheroid ASC groups differed (Fig. 2F).

\section{Effects of injected ASCs on the lungs of mice with elastase-induced emphysema}

The harvested lung tissues from mice with or without elastase-induced emphysema were analyzed by ELISA and Western blotting (data not shown) to identify changes in host cells after the transplantation of dissociated or spheroid ASCs. The protein levels of mouse FGF-2, HGF, and VEGF were quantified using ELISA. Mouse FGF-2 was significantly increased in the spheroid ASC-injected group when compared with the dissociated ASC-injected group (Fig. 3A). Mouse HGF and VEGF levels were not significantly different between dissociated and spheroid ASC-injected groups (Fig. 3B and C). The growth factor levels in the three treatment groups were not significantly different from the control (-) group.

Effects of injected ASCs on the regulation of MMP production To observe the effects of ASCs on tissue regeneration, the mRNA transcript levels of MMP-2, -9 , and -12 in lung tissue were measured by qPCR. MMP-2 mRNA expression tended to be lower in the injected ASC group when compared with the negative control group. MMP-12 expression was significantly lower in the spheroid ASC-injected group when compared with the dissociated ASC-injected group (Fig. 4A). Protein expression levels were evaluated by Western blotting (Fig. 4B) and zymography (Fig. 4C). MMP-9 expression at the mRNA and protein level was similar between the dissociated and spheroid ASC-injected groups. Both groups also showed reduced MMP-2 expression and activity. Additionally, the spheroid ASC-injected group showed a greater reduction in MMP-2 activity when compared with the dissociated ASC injected group. To extend these findings, we tested the expression levels of tissue inhibitor of metalloproteinase-1 (TIMP-1) and secretory leukocyte protease inhibitor (SLPI), which both inhibit MMPs (Fig. 4D). Surprisingly, the injection of either dissociated or spheroid ASCs significantly increased the mRNA transcript levels of TIMP-1, which encodes a protease inhibitor. The transplantation of spheroid ASCs resulted in a greater increase in TIMP-1 and SLPI expression when compared with dissociated ASCs.
A

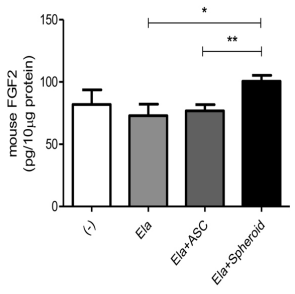

B

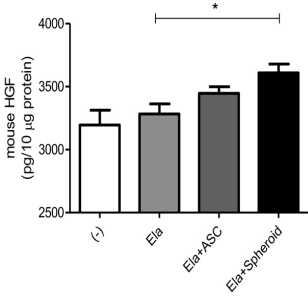

C

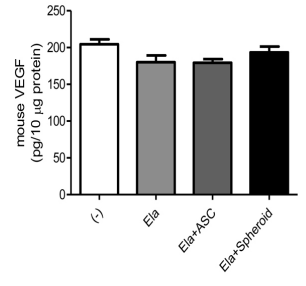

A

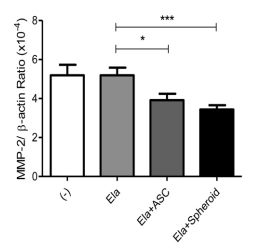

B

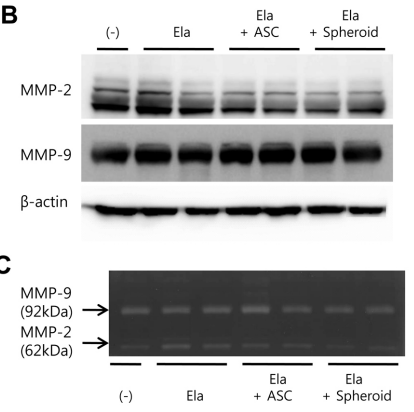

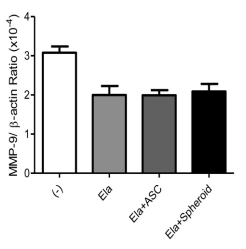

D

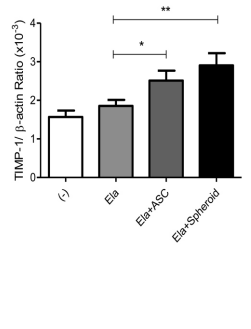

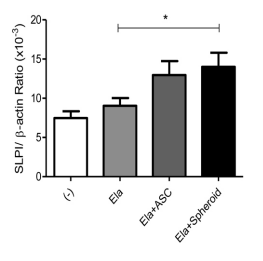

Fig. 3. Growth factor production in lungs with emphysema: (A) FGF-2, (B) $\mathrm{HGF}$, and (C) VEGF. ${ }^{*} \mathrm{P}<0.05$ and $* * P<0.01$ for comparisons between 2 groups.
Fig. 4. Effects of ASCs on MMPs, TIMP-1 and SLPI expression in lungs with emphysema. (A) The mRNA levels of MMP-2, MMP-9, and MMP-12 in the lung. (B) and (C) Representative images for western blotting and gelatin zymography. (D) The mRNA expression of TIMP-1 and SLPI. $* P<0.05$, $* * P<0.01$, and $* * * P<0.001$ for comparisons between 2 groups. 


\section{DISCUSSION}

In the current study, we examined if spheroid ASCs showed improved therapeutic efficacy in a mouse elastase-induced model of emphysema. Classically, MSCs have been cultured as a two-dimensional (2D) monolayer using coated culture plates. However, 2D cultures are disadvantaged by the fact that MSCs lose their stemness properties (e.g., replication potential and differentiation capacity) when grown in this manner; notably, this phenomenon does not occur in humans or mice in vivo (14). To overcome this problem, many researchers have tried to adopt three-dimensional (3D) spheroid cultures. The effect of spheroid formation on cellular differentiation and cell-cell interactions has been studied. The formation of a spheroid prevented apoptosis, and thus facilitated differentiation, because of the conservation of cell-cell interactions that were important for survival and colony formation $(15,16)$.

Here we compared the expression of apoptotic and growth factors between dissociated and spheroid ASCs. A previous report showed that spheroid ASCs decreased BAX protein expression when compared with dissociated ASCs (17). Our Western blot results showed no significant difference in BAX protein levels and increased $\mathrm{BCl}-2$ protein expression in spheroid ASCs when compared with dissociated ASCs. The correlation of $\mathrm{BAX}$ and $\mathrm{BCl}-2$ expression was not determined.

Growth factors perform an important role in tissue regeneration. FGF-2 is a basic fibroblast growth factor involved in multiple biological activities, including angiogenesis, migration, proliferation, and anti-apoptosis (18-20). VEGF is involved in vascular regulation in angiogenesis, cell-cell and cell-matrix interactions, and the proliferation of endothelial cells in injured lung tissue. VEGF was reported as a crucial regulatory factor in the aggravation or recovery of emphysema (21-23). We observed an increase in FGF-2 and VEGF in cells and tissues without a release of FGF2 in the culture medium.

In various animal models, the major advantages of spheroid MSCs are their higher level of stemness and anti-inflammatory properties and exertion of stronger therapeutic effects, such as increased growth factor secretion in the recipient and greater differentiation capacity $(13,16,17,24)$. We did not assess stemness in our current study; however, the effects of injected spheroid ASCs were confirmed by the increased growth factor expression in the host tissue when compared with dissociated ASCs.

The use of spheroid ASCs in our current study significantly attenuated emphysema in a mouse elastase-induced model when compared with dissociated ASCs at a similar dose of $1 \times$ $10^{5}$ cells. To characterize this therapeutic mechanism, we measured growth factor and MMP expression in mouse lung tissue lysates.

The expression of each growth factor was significantly enhanced, and the expression of MMPs (-2 and -12) was diminished when compared with the dissociated ASC-injected group. In contrast, the expression and activity of the protease inhibitors TIMP-1 and SLPI have significantly enhanced in spheroid ASC-injected mice. Previous studies revealed that exogenous growth factors, i.e., EGF, VEGF, and HGF as an angiogenic factor, enhanced the expression of MMPs in vitro and in vivo via cell migration and proliferation and ECM remodeling (25-27). The MMPs $(-2,-9$, and -12$)$ assayed in our current analyses are proteases with major roles in emphysema. These MMPs are secreted by various cells, including epithelial cells, neutrophils, eosinophils, and alveolar macrophages, and they can degrade matrix components in normal and abnormal states (28). MMP-12 helps macrophages to invade tissues and thus contributes to the degradation of the alveolar wall. In a recent report, the pathogenesis of emphysema was blocked at an early stage by ablating the MMP-12 gene or inhibiting MMP-9 and MMP-12 in a smoke-induced emphysema animal model (29-31). TIMP-1 inhibits the activity of most MMPs, including MMP-12, but not the membrane-type MMPs $(-14$, $-15,-16,-17)$, whereas SLPI inhibits the activity of elastase (32). Some reports have shown that proteins secreted by MSCs can protect tissues by inhibiting the activity of endogenous and exogenous MMPs and proteases via the ERK1/2 pathway and other proteins, such as erythropoietin, TIPMs, and VEGF in vitro $(2,33,34)$.

Our current findings suggest that dissociated or spheroid ASCs injected in a mouse elastase-induced model of emphysema inhibit MMPs via TIMP-1 and SLPI and induce regeneration via growth factor production. Moreover, the use of spheroid cells resulted in an improved therapeutic efficacy in vivo when compared with dissociated cells.

Spheroid ASCs enhanced recovery in an elastase-induced mouse model of emphysema by increasing growth factor production and anti-proteases when compared with dissociated ASCs at the same dose. Thus, spheroid ASCs have potential as a future treatment of emphysema.

\section{MATERIALS AND METHODS}

\section{Mice}

Pathogen-free female C57BL/6 mice were used at 6 weeks of age and with each one having a body weight of $20 \mathrm{~g}$ (Seongnam, Korea). All animals were cared for according to the guidelines of the Institutional Animal Care and Use Committee of Asan Medical Center (Seoul, Korea).

\section{ASC culture}

Human ASCs were purchased from Invitrogen (Carlsbad, CA, USA). ASCs were cultured in MesenPRO RS Medium supplemented with Growth Supplement (Invitrogen, CA, USA) and $1 \%$ penicillin at $37^{\circ} \mathrm{C}$ in a $5 \% \mathrm{CO}_{2}$ incubator. ASCs at passage 5 were used for all experiments.

\section{Spheroid formation}

Spheroids were formed using polydimethylsiloxane (PDMS)based concave microwell molds. Concave microwells with a 
diameter of $300 \mu \mathrm{m}$ were coated with $70 \%(\mathrm{v} / \mathrm{v})$ ethanol, followed by PBS and 3\% (W/V) BSA to prevent cell attachment. Each concave microwell contained 64 holes and suspended ASCs were seeded at a density of $1 \times 10^{5}$ per well. After several minutes, the remaining cells were removed using ASC culture media with pipette. Concave micro-wells seeded with ASCs were incubated at $37^{\circ} \mathrm{C}$ in a $5 \% \mathrm{CO}_{2}$ incubator for $24 \mathrm{~h}$.

\section{An elastase-induced mouse model of emphysema and ASC transplantation}

C57BL/6 mice were anesthetized intraperitoneally (i.p.) with $16 \mu$ of Zoletil 50 (Virbac Laboratories, Carros, France) and 4 $\mu$ l of Rompun (Bayer Korea, Ansan, Korea). Next, mice were intratracheally (i.t.) administered porcine pancreatic elastase $(0.4 \mathrm{U}, 8 \mathrm{U} / \mathrm{ml}$; Sigma-Aldrich, St. Louis, MO, USA) at day 0. After 7 days, mice were intrapleurally injected with dissociated or spheroid ASCs $\left(1 \times 10^{5}\right.$ cells $)$ using a syringe equipped with a 26-gauge needle.

\section{Gene expression analysis by quantitative polymerase chain reaction (qPCR)}

Total RNAs from lung tissues and ASCs were extracted using an RNeasy Mini Kit (Qiagen, Düsseldorf, Germany) and synthesized into cDNA using a cDNA synthesis kit (Thermo Fisher Scientific, Waltham, MA, USA). qPCR analyses were performed with a real-time LightCycler 480 with SYBR Green I master mix (Roche Diagnostics, CA, USA) and primers. The list of primers used is shown in Supplementary Table 1.

\section{Detection of MMP activity assays using zymography}

To detect MMP activity in the mouse lung, tissues were homogenized in a radioimmunoprecipitation assay buffer (RIPA; Cell Signaling Technology, Danvers, MA, USA) without a protease inhibitor and quantified using a BCA kit (Thermo Scientific, CA, USA). Samples containing $10 \mu \mathrm{g}$ of protein were subjected to $10 \%$ SDS-PAGE using a gelatin-based gel (Sigma-Aldrich, MO, USA). To develop the zymogram, the gels were incubated in $1 \times$ zymogram development buffer (Bio-Rad, CA, USA) at $37^{\circ} \mathrm{C}$ for $12 \mathrm{~h}$. Next, gels were stained with $0.5 \%$ Coomassie blue R-250 (Bio-Rad, CA, USA) in 30\% ethanol and $10 \%$ acetic acid for $1 \mathrm{~h}$. Destaining was performed with $30 \%$ ethanol and $10 \%$ acetic acid.

\section{Enzyme-linked immunosorbent assay (ELISA)}

A total of $10 \mu \mathrm{g}$ of protein from mouse tissue and $30 \mu \mathrm{g}$ of total protein from $1 \times 10^{6}$ ASCs were used for ELISAs (R\&D Systems; Minneapolis, MN) according to the manufacturer's instructions. The measured proteins included human and mouse growth factors (FGF-2, HGF, and VEGF).

\section{Western blotting}

Lysates of tissues and cells were homogenized in RIPA buffer and quantified using the BCA assay. Thirty microgram samples were separated by SDS-PAGE using a 10-15\% gel and then transferred to a PVDF membrane. Primary antibodies against BAX, BCl-2, FGF2, VEGF, MMP-2, and MMP-9 (Cell Signaling Technology, Danvers, MA) and their corresponding secondary antibodies were incubated with the membrane overnight at $4^{\circ} \mathrm{C}$ and for $1 \mathrm{~h}$ at room temperature, respectively. Membranes were scanned with LAS 4000 (Fujifilm; Tokyo, Japan) using film to detect the protein signals.

\section{Histology and quantification of emphysema}

The histology of the lungs was completed using a previously described method (8). Briefly, the perfused lungs were inflated with $0.5 \%$ low-melting agarose, fixed with $4 \%$ formalin, and embedded in paraffin. Lung sections with a thickness of $6 \mu \mathrm{m}$ were stained with $\mathrm{H}$ and $\mathrm{E}$. The mean linear intercepts (MLI) were determined from the microscopic images.

\section{Data analysis}

Statistical analyses were performed using the GraphPad Prism ver. 5 (GraphPad software, La Jolla, CA, USA). The data are presented as the mean \pm SEM. The Mann-Whitney test was used to compare groups, and statistical significance was set at $\mathrm{P}<0.05$.

\section{ACKNOWLEDGEMENTS}

The authors thank the members of the Asan Medical Center animal facility for their technical expertise.

This work was supported by the Korean Health Technology R\&D Project, Ministry of Health \& Welfare, Republic of Korea [grant number HI14C1487] and the National Research Foundation of Korea [grant number NRF-2015K1A4A3046807].

\section{CONFLICTS OF INTEREST}

Authors have no conflicting financial interests.

\section{REFERENCES}

1. Lange P, Marott JL, Vestbo J et al (2012) Prediction of the clinical course of the chronic obstructive pulmonary disease, using the new GOLD classification: a study of the general population. Am J Respir Crit Care Med 186, 975-981

2. Wang $Y$, Hu X, Xie X, He A, Liu X and Wang JA (2011) Effects of mesenchymal stem cells on matrix metalloproteinase synthesis in cardiac fibroblasts. Exp Biol Med (Maywood) 236, 1197-1204

3. Churg A, Zhou S and Wright JL (2012) Series "matrix metalloproteinases in lung health and disease": Matrix metalloproteinases in COPD. Eur Respir J 39, 197-209

4. Park IS, Rhie JW and Kim SH (2014) A novel threedimensional adipose-derived stem cell cluster for vascular regeneration in ischemic tissue. Cytotherapy $16,508-522$

5. Zhao YF, Xiong W, and Wu XL (2014) Mesenchymal stem cell-based developmental endothelial locus-1 gene therapy for acute lung injury induced by lipopolysaccharide in 
mice. Mol Med Rep 9, 1583-1589

6. Guan XJ, Song L, Han FF et al (2013) Mesenchymal stem cells protect cigarette smoke-damaged lung and pulmonary function partly via VEGF-VEGF receptors. J Cell Biochem 114, 323-335

7. Rojas M, Xu J, Woods CR et al (2005) Bone marrowderived mesenchymal stem cells in repair of the injured lung. Am J Respir Cell Mol Biol 33, 145-152

8. Kim YS, Kim JY, Shin DM, Huh JW, Lee SW and Oh YM (2014) Tracking intravenous adipose-derived mesenchymal stem cells in a model of elastase-induced emphysema. Tuberc Respir Dis (Seoul) 77, 116-123

9. Terrovitis J, Lautamaki R, Bonios M et al (2009) Noninvasive quantification and optimization of acute cell retention by in vivo positron emission tomography after intramyocardial cardiac-derived stem cell delivery. J Am Coll Cardiol 54, 1619-1626

10. Zhang $M$, Methot D, Poppa $V$, Fujio $Y$, Walsh $K$ and Murry CE (2001) Cardiomyocyte grafting for cardiac repair: graft cell death and anti-death strategies. J Mol Cell Cardiol 33, 907-921

11. Yoon HH, Bhang SH, Shin JY, Shin J and Kim BS (2012) Enhanced cartilage formation via three-dimensional cell engineering of human adipose-derived stem cells. Tissue Eng Part A 18, 1949-1956

12. Cheng NC, Wang S and Young TH (2012) The influence of spheroid formation of human adipose-derived stem cells on chitosan films on stemness and differentiation capabilities. Biomaterials 33, 1748-1758

13. Bartosh TJ, Ylostalo JH, Mohammadipoor A et al (2010) Aggregation of human mesenchymal stromal cells (MSCs) into 3D spheroids enhances their anti-inflammatory properties. Proc Natl Acad Sci U S A 107, 13724-13729

14. Okumura K, Nakamura K, Hisatomi Y et al (2003) Salivary gland progenitor cells induced by duct ligation differentiate into hepatic and pancreatic lineages. Hepatology 38, 104-113

15. Bates RC, Buret A, van Helden DF, Horton MA and Burns GF (1994) Apoptosis induced by inhibition of intercellular contact. J Cell Biol 125, 403-415

16. Yamaguchi $\mathrm{Y}$, Ohno J, Sato A, Kido $\mathrm{H}$ and Fukushima $\mathrm{T}$ (2014) Mesenchymal stem cell spheroids exhibit enhanced in-vitro and in-vivo osteoregenerative potential. BMC Biotechnol 14, 105

17. Park IS, Chung PS and Ahn JC (2014) Enhanced angiogenic effect of adipose-derived stromal cell spheroid with low-level light therapy in hind limb ischemia mice. Biomaterials 35, 9280-9289

18. Ware JA and Simons M (1997) Angiogenesis in ischemic heart disease. Nat Med 3, 158-164

19. Yanagisawamiwa A, Uchida $Y$, Nakamura $F$ et al (1992) Salvage of Infarcted Myocardium by Angiogenic Action of Basic Fibroblast Growth-Factor. Science 257, 1401-1403

20. Scholz D, Cai WJ and Schaper W (2001) Arteriogenesis, a new concept of vascular adaptation in occlusive disease.
Angiogenesis 4, 247-257

21. Shigemura N, Sawa Y, Mizuno S et al (2005) Amelioration of pulmonary emphysema by in vivo gene transfection with hepatocyte growth factor in rats. Circulation 111, 1407-1414

22. Zeng $X$, Wert SE, Federici R, Peters KG and Whitsett JA (1998) VEGF enhances pulmonary vasculogenesis and disrupts lung morphogenesis in vivo. Dev Dyn 211, 215-227

23. Kanazawa H (2007) Role of vascular endothelial growth factor in the pathogenesis of the chronic obstructive pulmonary disease. Med Sci Monit 13, Ra189-Ra195

24. Zhang S, Liu P, Chen L, Wang Y, Wang Z and Zhang B (2015) The effects of the spheroid formation of adiposederived stem cells in a microgravity bioreactor on stemness properties and therapeutic potential. Biomaterials 41, 15-25

25. Wang $\mathrm{H}$ and Keiser JA (1998) Vascular endothelial growth factor upregulates the expression of matrix metalloproteinases in vascular smooth muscle cells - Role of flt-1. Circ Res 83, 832-840

26. Wang $\mathrm{H}$ and Keiser JA (2000) Hepatocyte growth factor enhances MMP activity in human endothelial cells. Biochem Biophys Res Commun 272, 900-905

27. Seandel M, Noack-Kunnmann K, Zhu D, Aimes RT and Quigley JP (2001) Growth factor-induced angiogenesis in vivo requires specific cleavage of fibrillar type I collagen. Blood 97, 2323-2332

28. Loffek S, Schilling O and Franzke CW (2011) Series "matrix metalloproteinases in lung health and disease": Biological role of matrix metalloproteinases: a critical balance. Eur Respir J 38, 191-208

29. Shipley JM, Wesselschmidt RL, Kobayashi DK, Ley TJ and Shapiro SD (1996) Metalloelastase is required for macrophage-mediated proteolysis and matrix invasion in mice. Proc Natl Acad Sci U S A 93, 3942-3946

30. Churg A, Cosio M and Wright JL (2008) Mechanisms of cigarette smoke-induced COPD: insights from animal models. Am J Physiol Lung Cell Mol Physiol 294, L612-631

31. Churg A, Wang R, Wang X, Onnervik PO, Thim K and Wright JL (2007) Effect of an MMP-9/MMP-12 inhibitor on smoke-induced emphysema and airway remodeling in guinea pigs. Thorax 62, 706-713

32. Visse $R$ and Nagase $H$ (2003) Matrix metalloproteinases and tissue inhibitors of metalloproteinases: structure, function, and biochemistry. Circ Res 92, 827-839

33. Lozito TP and Tuan RS (2011) Mesenchymal stem cells inhibit both endogenous and exogenous MMPs via secreted TIMPs. J Cell Physiol 226, 385-396

34. Gruber R, Kandler B, Holzmann P et al (2005) Bone marrow stromal cells can provide a local environment that favors migration and formation of tubular structures of endothelial cells. Tissue Eng 11, 896-903 